\title{
Influencia de cinco tipos de pastas térmicas en el rendimiento de tres modelos de computadoras personales
}

\section{Influence of five types of thermal pastes on the performance of three personal computer models}

\author{
Lineth Guerra ${ }^{l}$, Cirilo Castro ${ }^{l}$ José Mendoza ${ }^{1 *}$ \\ Licenciatura en Desarrollo de Software, Centro Regional de Bocas del Toro, Universidad Tecnológica de Panamá
}

\begin{abstract}
Resumen Este trabajo analiza la influencia de cinco tipos de pastas térmicas en el rendimiento de tres modelos de computadoras personales. Se aplicó un enfoque de investigación cuantitativo, el diseño fue no experimental. La población de estudio fueron tres computadoras personales a las cuales se les cambió el compuesto térmico por cinco tipos diferentes de pastas térmicas. La investigación se realizó en el distrito de Changuinola, provincia de Bocas del Toro, Panamá. Los resultados indican que, con la pasta térmica a base de plata se alcanzó una alta conductividad térmica, al tiempo que se adoptó al coeficiente de expansión térmica (CTE), logrando aumentar significativamente la capacidad térmica del disipador para deshacer el calor generado por los microprocesadores. Por lo tanto, se concluyó que, la selección de la pasta térmica es una variable para tomar en cuenta a la hora de seleccionar el compuesto apropiado para el procesador. El estudio reflejó que, para las condiciones ambientales de Bocas del Toro, Panamá, la pasta térmica con mejor rendimiento de las cinco pruebas, fue la del compuesto a base de plata.
\end{abstract}

Palabras clave Procesador, pasta térmica, temperatura.

\begin{abstract}
This paper analyzes the influence of five types of thermal pastes on the performance of three personal computer models. A quantitative research approach was applied, the design was non-experimental. The study population was three personal computers to which the thermal compound was exchanged for five different types of thermal pastes. The investigation was conducted in the district of Changuinola, province of Bocas del Toro, Panama. The results indicate that, with the silver-based thermal paste, a high thermal conductivity was reached, while the coefficient of thermal expansion (CTE) was adopted, achieving a significant increase in the thermal capacity of the dissipater to undo the heat generated by the microprocessors. Therefore, it was concluded that, the selection of the thermal paste is a variable to take into account when selecting the appropriate compound for the processor- The study showed that, for the environmental conditions of Bocas del Toro, Panama, the thermal paste with better performance of the five tests, was that of the silver-based compound.
\end{abstract}

Keywords Processor, thermal paste, temperature.

*Corresponding author: jose.mendoza@utp.ac.pa

\section{Introducción}

Desde la creación de la placa madre AT Advanced Technology, figura 1, en el año 1984 por IBM con procesador Intel [1], la temperatura que generaba el procesador representaba el inicio de un gran problema para los componentes de la placa base. Por eso se empezaron a implementar los sistemas de enfriamiento por disipadores de calor y con ello inicia el uso e implementación de las pastas térmicas, ya que estas son las que hacen posible la correcta transferencia de la temperatura desde el procesador hasta el disipador. El uso de este sistema de enfriamiento tomó más auge con el surgimiento de las ATX Advanced Technology Extended en el año 1997 porque los procesadores, al ser más potentes y con mayor número de transistores que sus antecesores, generaban una temperatura aún más elevada [2]. Se consideró oportuno investigar este tema, ya que las actualizaciones de las tecnologías han generado una cantidad grande de variantes en cuanto a disipadores de calor, y las soluciones térmicas también han evolucionado desde las almohadillas térmicas hasta las pastas térmicas a base de metal líquido [3].

Los compuestos térmicos que se comenzaron a utilizar fueron las almohadillas térmicas, ya que era mínima la generación de temperatura en esos tiempos; luego pasaron a las pastas de cerámica. Con el aumento de las temperaturas se cambiaron los compuestos de la pasta hasta el punto de que, hoy en día contienen diminutas partículas de oro, plata, aluminio o cobre que deben utilizarse con un disipador 
apropiado para no causar un daño irreversible al microprocesador.

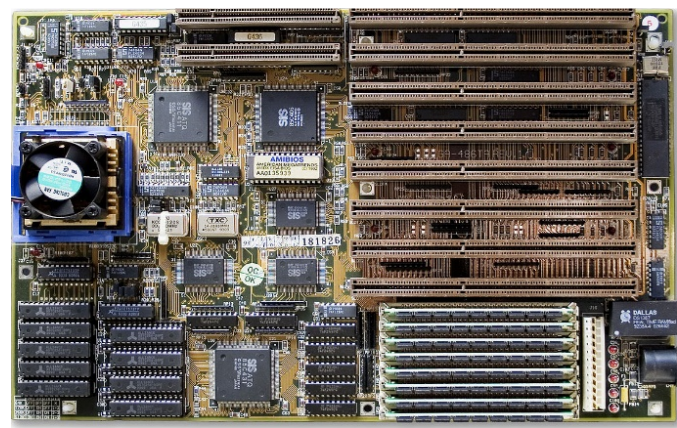

Figura 1. Placa Madre AT 1984.

Fuente: [1].

Los dispositivos semiconductores son cada vez más pequeños y más densos con la evolución de las nuevas tecnologías. Sin embargo, los aumentos en la densidad del circuito producen un énfasis correspondiente en las estrategias generales de encapsulado de los chips, para seguir siendo competitivos. Por lo tanto, los fabricantes de chips y sustratos, para encapsulados, se ven constantemente desafiados a mejorar la calidad de sus productos, identificando y eliminando problemas, reduciendo el tamaño y el peso de estos, a un costo más bajo, proporcionando eficiencias térmicas mejoradas y más avanzadas [4].

En los últimos años, la tecnología del diseño de disipador de calor con aletas ha alcanzado las técnicas comunes para la refrigeración electrónica [5], como se muestra en la figura 2.

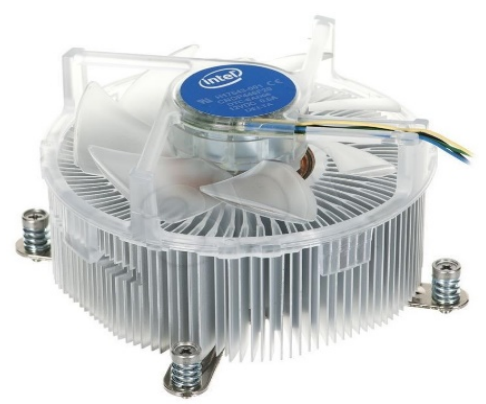

Figura 2. Solución térmica Intel BXTS13A disipador. Fuente: Intel [6].

El disipador de calor que se usa en los procesadores de las computadoras personales es una fusión de un conjunto de materiales de alta conductividad térmica, tales como cobre, aluminio, latón, cobre/níquel, aluminio/latón, se usan para fabricar disipadores de calor con aletas (FHS - Flower Heat Sinks) dependiendo del costo y la simplicidad de fabricación de estos materiales.
El sistema de enfriamiento de los procesadores modernos se basa en un disipador de calor totalmente metálico; el cual posee un diseño optimizado de disipación de calor en base de cobre o aluminio con aletas de aluminio unidas, y su función se basa en las leyes de enfriamiento de Newton y la transferencia de calor [7].

El comportamiento de la temperatura en el disipador de calor se da como se muestra en la figura 3.

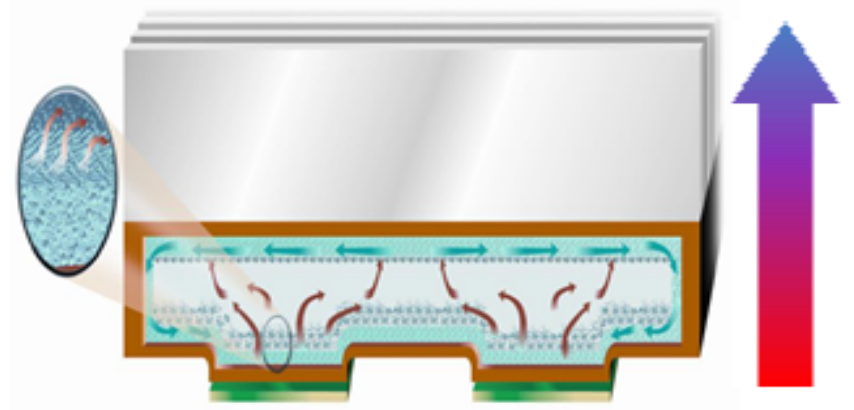

Figura 3. Dirección que recorre la temperatura en el disipador. Fuente: [8].

El objetivo general de la investigación fue demostrar la influencia de cinco tipos de pastas térmicas en el rendimiento de tres modelos de computadoras personales. La idea surgió al apoyar a un colegio que tenía escasez de equipo, al recuperar 18 máquinas que las consideraban dañadas y uno de los causales, era por sobrecalentamiento, al reemplazar la pasta térmica, se lograron recuperar algunas.

De esta anécdota de motivación, nace la pregunta problema ¿Cuál es el impacto de las pastas térmicas en la temperatura del procesador la computadora y cómo saber cuál es la más conveniente para aumentar la vida útil del procesador?

Se identifica la ausencia del conocimiento sobre el uso de las pastas térmicas y la importancia de conocer la función de estas. Se propone realizar pruebas para demostrar qué pasta térmica es la más eficiente en el proceso de transferencia de calor.

\section{Materiales y métodos}

La población de estudio fue tres computadoras personales a las cuales se les cambió las pastas térmicas. La investigación se realizó en el distrito de Changuinola, provincia de Bocas del Toro. La duración de la investigación fue de tres meses.

En el experimento se utilizaron cinco tipos de pastas térmicas en tres computadoras: una PC de escritorio con placa madre AsRock y dos computadoras personal (PC) portátiles marcas Asus y Acer, como se muestra en la figura 4. 
Mendoza (et al): Influencia de cinco tipos de pastas térmicas en el rendimiento de tres modelos de computadoras personales

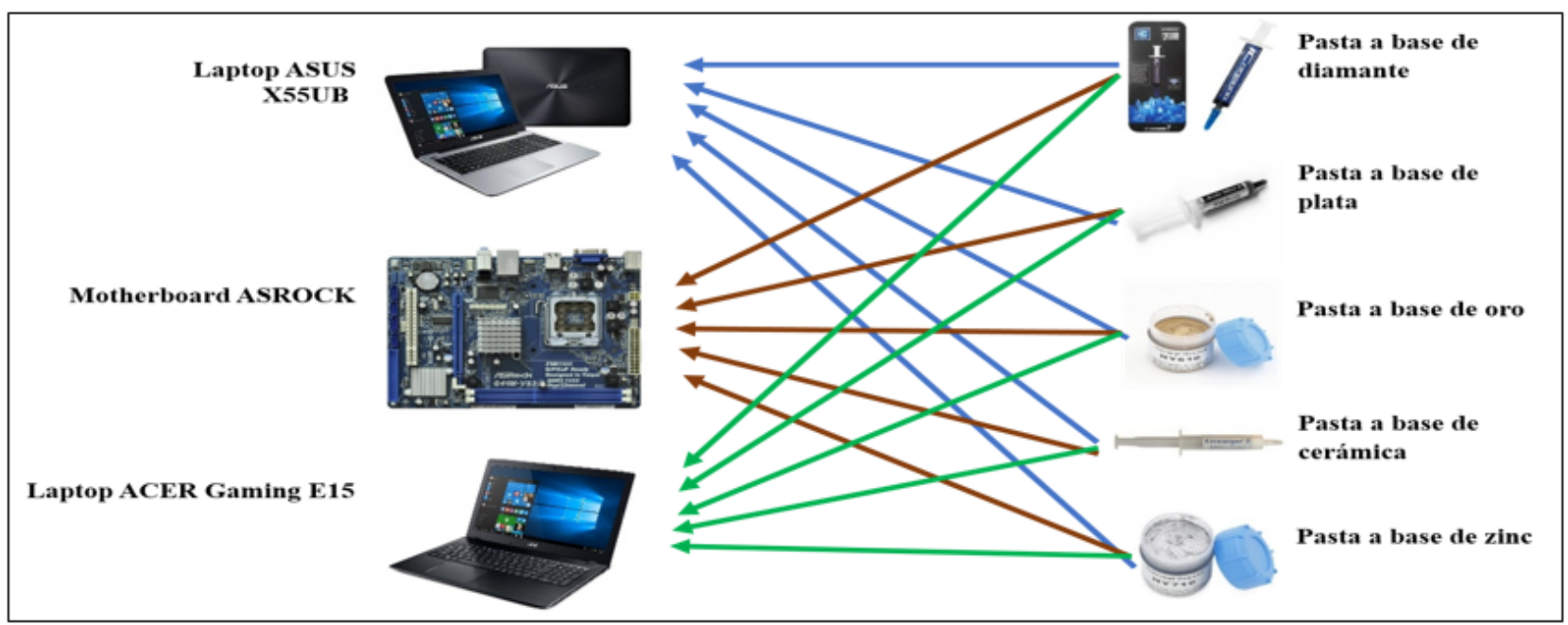

Figura 4. Diagrama de la aplicación de las cinco pastas térmicas con las computadoras.

El tipo de la investigación tiene un enfoque cuantitativo, el diseño de la investigación fue no experimental, porque no se manipulan las variables del estudio, por otro lado, también fue transversal. Los datos recolectados fueron analizados estadísticamente con Microsoft Excel, comparando las muestras entre sí, para emitir conclusiones.

\subsection{Variable: las pastas térmicas}

Para que la trasferencia entre el procesador y el disipador de calor sea efectiva, se necesita de un compuesto químico térmico, tipo adherente y removible.

El compuesto térmico, también conocido como pasta térmica y grasa térmica, es un material utilizado para rellenar los espacios microscópicos entre la unidad central de procesamiento (CPU) de una computadora y su disipador de calor. El compuesto térmico aumenta significativamente la capacidad del disipador de calor para enfriar la CPU, lo que permite que la CPU funcione a mayor velocidad y mejore el rendimiento del sistema. Algunos procesadores se quemarán y dejarán de funcionar sin este compuesto térmico [9].

Hay docenas de marcas de pasta térmica disponibles, el precio normalmente se establece en función de la conductividad térmica y la cantidad de compuesto en el tubo (generalmente en el rango de 3g, que es de algunos usos). Un tubo de $8.5 \mathrm{~W} / \mathrm{mK}$ compuesto a base de carbono, que es resistente al envejecimiento, cuesta alrededor de $\$ 10$ por $4 \mathrm{~g}$.

El tipo de compuesto se enumera normalmente como un material basado en metal tipo plata, diamante/carbono (a menudo llamado "diamante nano") o cerámica. Los compuestos a base de metal, como el compuesto de plata, usan diminutos copos de metal para ayudar a conducir el calor al frío. Los compuestos de diamante suelen ser un poco más duros provenientes del tubo, lo que requiere un trabajo adicional para dispensarlos, pero son teóricamente más fuertes durante largos períodos de uso [10].

\subsubsection{Clasificación de las pastas térmicas}

Hay tres tipos de materiales de interfaz térmica (TIM) que, podría encontrar en el mercado: estos son los basados en metales, cerámicos y los basados en silicio [11].

A continuación, hay una lista de material de interfaz térmica (TIM), sus pros y contras:

\section{- Basado en metal}

Pros - Ellos son los más populares en el mercado. También tienen el más alto nivel de conductividad térmica. El hecho de que en su mayoría son de metal es lo que los hace más eficientes en la conducción de calor.

Contras - Por otro lado, estas partículas de metal son eléctricamente conductoras, lo que representa una gran amenaza para los componentes eléctricos.

\section{- Basado en cerámica}

Pros - Están compuestos de partículas cerámicas, lo que significa que no son tan eléctricamente conductores como los TIM basados en metales.

Contras - No son tan conductores como sus contrapartes basadas en metales. La diferencia de temperatura varía alrededor de uno a tres grados centígrados.

\section{- Basado en silicio}

Pros - Simplemente vienen en los disipadores de calor y funcionan bien con el uso regular de la computadora.

Contras - No son muy eficientes en la conducción de calor. Muestran un bajo rendimiento de conductividad en comparación con los equivalentes de cerámica y metal. No es 
aconsejable para los constructores que planean sobre acelerar el procesador (overclocking).

\subsubsection{Tipos de pastas térmicas}

Tipos de pastas térmicas que, se llaman por muchos nombres y a continuación se encuentran entre los más comunes (ver figuras de la 5 a la 9):

- Pasta térmica metálica con base diamante.

- Pasta térmica metálica con base plata.

- Pasta térmica metálica con base oro y cobre.

- Pasta térmica metálica con base aluminio.

- Pasta térmica de cerámica [3].

En comparación con los metales convencionales, algunos materiales compuestos pueden lograr una conductividad térmica relativamente alta al tiempo que se adaptan al coeficiente de expansión térmica (CTE) cambiando su composición. Estos materiales compuestos consisten en una matriz de metal, cerámica o polímero y pueden cargarse con grafito de alta conductividad térmica o partículas de diamante y / o grafito [12].

\subsubsection{Pastas utilizadas en el experimento}

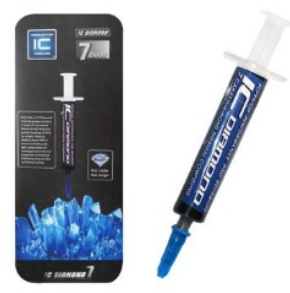

Figura 5. Pasta térmica metálico con base de diamante. Fuente: [14].

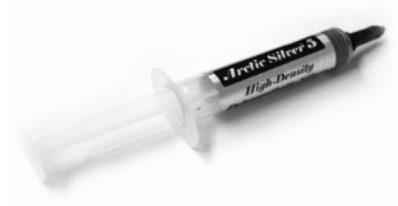

Figura 6. Pasta térmica metálico con base de plata. Fuente: [14].

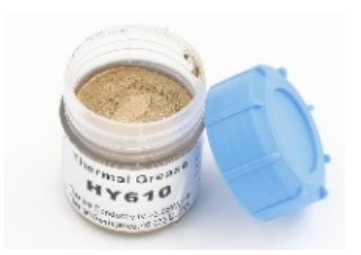

Figura 7. Pasta térmica metálico con base de oro y cobre. Fuente: [14].

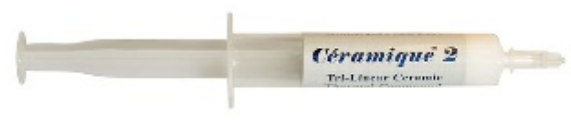

Figura 8. Pasta térmica metálico con base de cerámica. Fuente: [16].

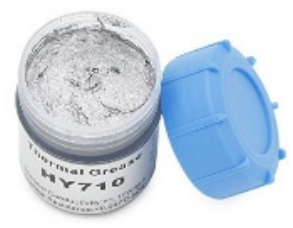

Figura 9. Pasta térmica metálico con base de zinc. Fuente: [14].

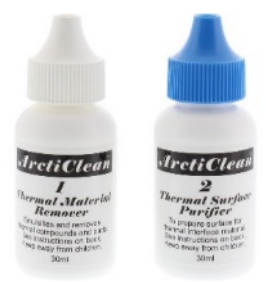

Figura 10. Líquidos para remover la pasta térmica. Fuente: [18].

\subsection{Variable temperatura}

La temperatura es un factor clave en el computador. Además, afecta de forma directa al rendimiento. A pesar de que el hardware está diseñado para soportar altas temperaturas de trabajo, si se les obliga a trabajar en estos límites térmicos durante demasiado tiempo acaba disminuyendo su rendimiento y fiabilidad [20].

Uno de los riesgos, aunque no se dañe el procesador, si trabaja a una temperatura demasiado alta, puede suceder una gran cantidad de errores y problemas de funcionamiento [21].

En los procesadores Intel, la empresa en el sitio de "Asistencia Técnica" cita: Estos términos y condiciones se refieren a la temperatura del procesador Intel para sistemas de escritorio y portátiles. El procesador no debe superar la temperatura máxima de chasis definida por el perfil térmico aplicable. El hecho de mantener la temperatura por debajo del máximo ayuda a optimizar el funcionamiento y la fiabilidad a largo plazo.

Para darle una mejor comprensión de las temperaturas de operación de los procesadores Intel y AMD, hemos incluido sus temperaturas en diferentes niveles de uso [22]:

- Temperatura de inactividad: computadora con el escritorio de Windows (sin abrir ventanas o programas).

- Temperatura normal: computadora durante el uso intensivo (juegos, edición de video, virtualización, etc.).

- Temperatura máxima: temperatura de CPU segura más alta recomendada por Intel o AMD.

Las marcas consideran que la temperatura de inactividad debe estar en el rango de 30 y 38 grados promedio. 


\subsection{Equipos utilizados en el experimento}

Para medir físicamente la temperatura se utilizó el termómetro infrarrojo de la pinza amperimétrica, se toma a menos de un metro de la superficie que se va a medir [22].

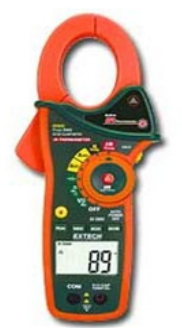

Figura 11. Pinza amperimétrica con termómetro de infrarrojos. Fuente: [14].

\subsection{Otros materiales de limpieza}

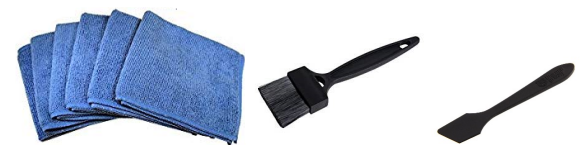

Figura 12. Paño de microfibra, brocha y espátula para aplicar la pasta.

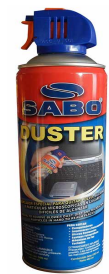

Figura 13. Aire comprimido removedor el polvo.

Fuente: [20].

\subsection{Equipos para evaluar}

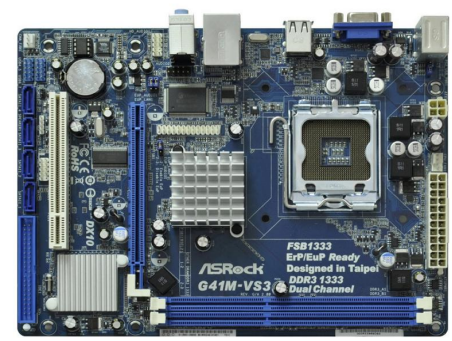

Figura 14. Placa madre ASRock G41M-VGS3.

Fuente: [15].

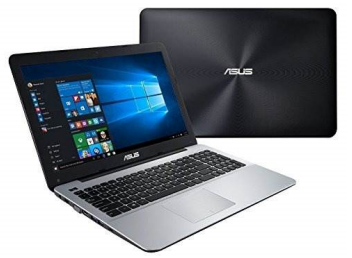

Figura 15. Laptop Asus X555UB.

Fuente: [16].

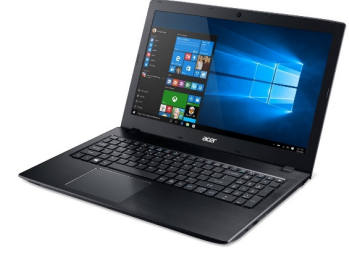

Figura 16. Laptop Acer Gaming E15.

Fuente: [17]

\section{Resultados y discusión}

Para la fase de prueba utilizamos el software Open Hardware para monitorear la temperatura del procesador, esta utilidad extrae los valores de la temperatura de los sensores de los núcleos de los microprocesadores, incluidos en el encapsulado de los fabricantes tanto Intel, como AMD. Además, es compatible con la mayoría de los chips de monitoreo de hardware que se encuentran en las placas madre modernas [14].

Se monitoreo físicamente con el sistema de medición de temperatura sin contacto de la pinza amperimétrica de $\mathrm{CA} / \mathrm{CC} /$ multímetro digital con termómetro de infrarrojos incorporado [14].

\subsection{Fase de prueba 1: placa madre ASRock}

La medición realizada de la placa madre ASRock G41MVGS3 [22], con procesador Intel Core 2, antes de cambiar la pasta térmica fue como se aprecia en la figura 17.

\begin{tabular}{|llll|}
\hline Temperatures & & & \\
CPU Core $\# 1$ & $40.0^{\circ} \mathrm{C}$ & $40.0{ }^{\circ} \mathrm{C}$ & $50.0{ }^{\circ} \mathrm{C}$ \\
CPU Core $\# 2$ & $48.0{ }^{\circ} \mathrm{C}$ & $48.0{ }^{\circ} \mathrm{C}$ & $52.0^{\circ} \mathrm{C}$ \\
\hline
\end{tabular}

Figura 17. Captura de pantalla de la temperatura de la placa madre ASRock G41M-VGS3, antes de cambiar la pasta térmica.

La tabla 1, muestra los resultados de las pruebas realizadas al procesador de la placa madre ASRock, después de haber aplicado las cinco pastas térmicas involucradas en la investigación.

Tabla 1. Temperatura registrada del procesador de la placa madre ASRock

\begin{tabular}{ccc}
\multicolumn{3}{c}{ con las cinco (5) pastas térmicas } \\
\hline Tipo pasta & $\begin{array}{c}\text { Temperatura } \\
\text { registrada por } \\
\text { software }^{\circ} \mathbf{C}\end{array}$ & $\begin{array}{c}\text { Temperatura } \\
\text { registrada } \\
\text { físicamente }^{\circ} \mathbf{C}\end{array}$ \\
\hline Pasta de diamante & $35^{\circ}$ & $38^{\circ}$ \\
Pasta de oro & $38^{\circ}$ & $46^{\circ}$ \\
Pasta de cerámica & $61^{\circ}$ & $59^{\circ}$ \\
Pasta de plata & $3^{\circ}$ & $36^{\circ}$ \\
Pasta de zinc & $65^{\circ}$ & $60^{\circ}$ \\
\hline
\end{tabular}

Resultando con un mejor rendimiento la pasta a base de "plata", tal como se muestra en la figura 18. 


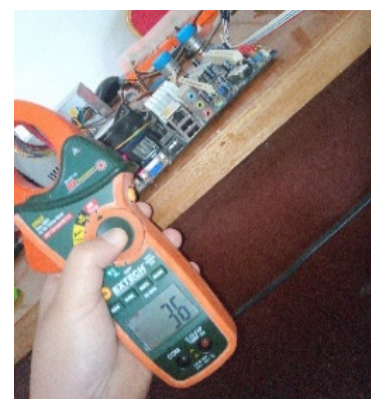

Figura 18. Tomando la temperatura física de la placa madre ASRock.

\subsection{Fase de prueba 2: computadora portátil Asus X555UB}

La temperatura registrada antes de cambiar la pasta térmica a la PC portátil marca Asus X555UB con procesador i5 6200u [16], fue la que se presenta en la figura 19.

\begin{tabular}{|cll|}
\hline Temperatures & & \\
CPU Core $\# 1$ & $48.0^{\circ} \mathrm{C}$ & $50.0^{\circ} \mathrm{C}$ \\
CPU Core \#2 & $48.0^{\circ} \mathrm{C}$ & $49.0^{\circ} \mathrm{C}$ \\
CPU Core $\# 3$ & $48.0^{\circ} \mathrm{C}$ & $49.0^{\circ} \mathrm{C}$ \\
CPU Core $\# 4$ & $49.0^{\circ} \mathrm{C}$ & $54.0^{\circ} \mathrm{C}$ \\
CPU Package & $49.0^{\circ} \mathrm{C}$ & $54.0^{\circ} \mathrm{C}$ \\
\hline
\end{tabular}

Figura 19. Captura de la pantalla de temperatura registrada de la portátil Asus X555UB, antes de hacer las pruebas con las pastas térmicas.

La tabla 2, muestra los resultados de las pruebas realizadas a la portátil Asus después de haber aplicado las cinco pastas térmicas involucradas en la investigación.

Tabla 2. Temperatura del procesador de la PC portátil Asus con las cinco (5) pastas térmicas

\begin{tabular}{ccc}
\hline Tipo pasta & $\begin{array}{c}\text { Temperatura } \\
\text { registrada por } \\
\text { software }{ }^{\circ} \mathbf{C}\end{array}$ & $\begin{array}{c}\text { Temperatura } \\
\text { registrada } \\
\text { físicamente }\end{array}$ \\
\hline Pasta de diamante & $45^{\circ}$ & $51^{\circ}$ \\
Pasta de oro & $55^{\circ}$ & $62^{\circ}$ \\
Pasta de cerámica & $40^{\circ}$ & $55^{\circ}$ \\
Pasta de plata & $36^{\circ}$ & $40^{\circ}$ \\
Pasta de zinc & $58^{\circ}$ & $60^{\circ}$ \\
\hline
\end{tabular}

Resultando con un mejor rendimiento la pasta a base de "plata". La figura 20 muestra el momento que se le aplicaba con la espátula la pasta térmica a la PC portátil Asus.

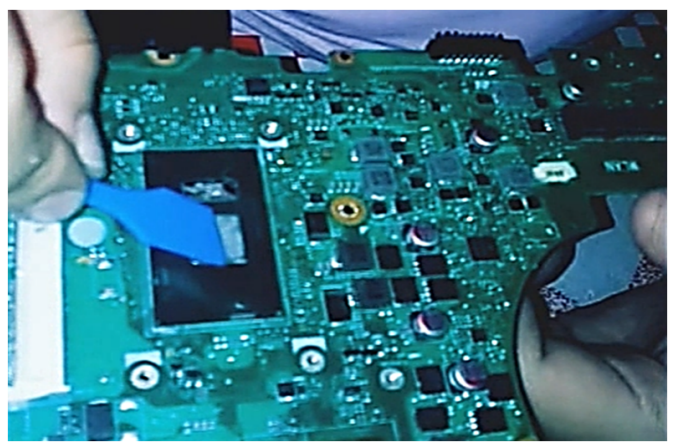

Figura 20. Aplicación de la pasta térmica al procesador de la PC portátil.

\subsection{Fase de prueba 3: computadora portátil Acer Gaming E15}

La temperatura registrada antes de cambiar la pasta térmica a la PC portátil marca Acer E15 con procesador i5 7200u [17], fue la que se presenta en la figura 21 .

\begin{tabular}{|ccc|}
\hline Temperatures & & \\
C... CPU Core $\# 1$ & $58.0^{\circ} \mathrm{C}$ & $68.0^{\circ} \mathrm{C}$ \\
... & $58.0^{\circ} \mathrm{C}$ & $70.0^{\circ} \mathrm{C}$ \\
CPU Core $\# 2$ & $60.0^{\circ} \mathrm{C}$ & $68.0^{\circ} \mathrm{C}$ \\
CPU Core $\# 3$ & $61.0^{\circ} \mathrm{C}$ & $71.0^{\circ} \mathrm{C}$ \\
CPU Core $\# 4$ & $61.0^{\circ} \mathrm{C}$ & $71.0^{\circ} \mathrm{C}$ \\
\hline
\end{tabular}

Figura 21. Captura de la pantalla de temperatura de la portátil Acer E15, antes de hacer las pruebas con las pastas térmicas.

La tabla 3, muestra los resultados de las pruebas realizadas con las cinco (5) pastas térmicas involucradas en la investigación.

\begin{tabular}{|c|c|c|}
\hline Tipo pasta & $\begin{array}{c}\text { Temperatura } \\
\text { registrada por } \\
\text { software }{ }^{\circ} \mathbf{C}\end{array}$ & $\begin{array}{c}\text { Temperatura } \\
\text { registrada } \\
\text { físicamente }{ }^{\circ} \mathrm{C}\end{array}$ \\
\hline Pasta de plata & $30^{\circ}$ & $34^{\circ}$ \\
\hline Pasta de zinc & $62^{\circ}$ & $59^{\circ}$ \\
\hline Pasta de oro & $75^{\circ}$ & $56^{\circ}$ \\
\hline Pasta de cerámica & $40^{\circ}$ & $62^{\circ}$ \\
\hline Pasta de diamante & $49^{\circ}$ & $75^{\circ}$ \\
\hline
\end{tabular}

Se puede observar que la pasta térmica que ofreció mejor resultado, con la temperatura más baja tanto por software Open Hardware, como por termómetro infrarrojo, fue la "pasta térmica a base de plata".

Para demostrar los resultados se graficó la fase de prueba 3 con la portátil Acer Gaming, resultando con un mejor rendimiento la pasta a "base de plata", en la fase de prueba 3 como se muestra en la figura 22 . 
Mendoza (et al): Influencia de cinco tipos de pastas térmicas en el rendimiento de tres modelos de computadoras personales

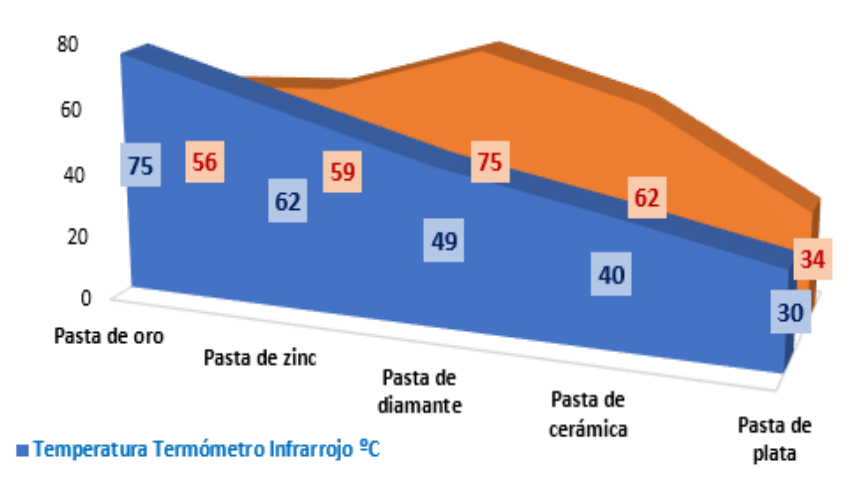

- Temperatura Termómetro Infrarrojo ${ }^{\circ} \mathrm{C}$

Figura 22. Gráfica de la temperatura del procesador de la PC Acer E15 con las cinco pastas térmicas.

La pasta térmica tiene una gran importancia e influye en la transferencia apropiada de calor del procesador al disipador de calor, como se observa en la figura 23.

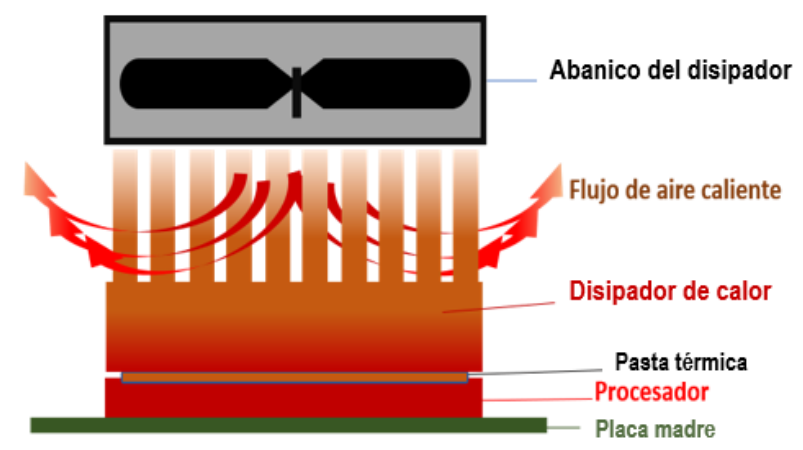

Figura 23. Flujo del aire que genera el abanico del disipador de calor.

Con la pasta térmica a base de plata se alcanzó una alta conductividad térmica, al tiempo que se adoptó al coeficiente de expansión térmica (CTE), logrando aumentar significativamente la capacidad térmica del disipador para deshacer el calor generado por los microprocesadores, como se muestra en la figura 24 .

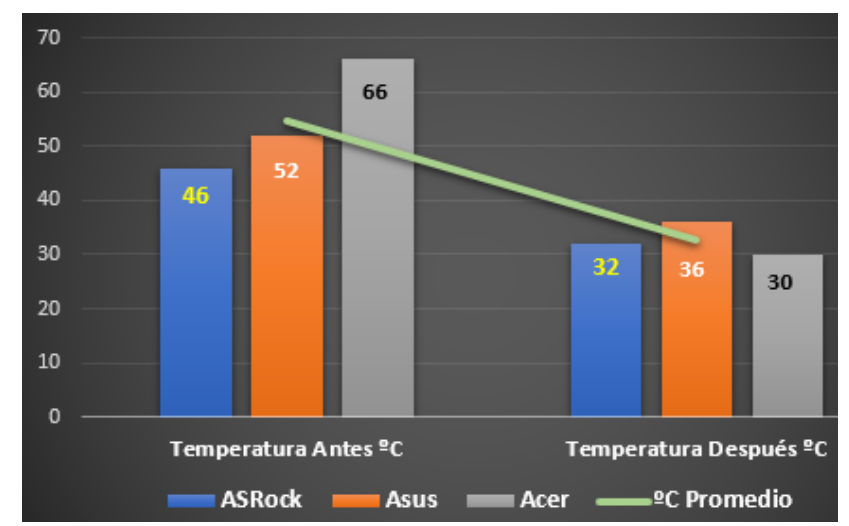

Figura 24. Gráfica de la variación de la temperatura de las tres computadoras antes y después de aplicar la pasta a base de plata.

\section{Conclusiones}

Con los resultados exhibidos en el presente artículo podemos concluir lo siguiente, las pastas térmicas juegan un papel importante en la transferencia térmica del procesador al disipador. Una pasta térmica dañada generaría altas temperaturas en el procesador bajando el rendimiento de la computadora hasta bloquearla, apagarla y finalmente causar daños irreversibles en el equipo.

Se recomienda a los usuarios estar pendientes de la temperatura de sus computadoras, y el equipo de tecnología, en especial al soporte técnico, monitorear a través de software la temperatura del procesador de las computadoras, para evitar futuros daños.

La selección de la pasta térmica es una variable para tomar en cuenta a la hora de seleccionar el compuesto apropiado para el procesador. El estudio reflejó que, para las condiciones ambientales de Bocas del Toro, Panamá, la pasta térmica con mejor rendimiento, en las cinco pruebas, fue la del compuesto a base de plata.

Se logró una conductividad térmica relativamente alta, al tiempo que, la pasta se adapta al coeficiente de expansión térmica (CTE), logrando aumentar significativamente la capacidad del disipador de calor para enfriar el microprocesador.

A continuación, se describe el interés de ampliar el trabajo a 50 computadoras, en los cuatro distritos de la provincia de Bocas del Toro, como trabajo final de grado.

\section{AGRADECIMIENTOS}

En primer lugar, agradecerle a nuestro profesor asesor por la motivación constante y asesoramiento en el desarrollo de la investigación y redacción del artículo.

\section{REFERENCIAS}

[1] [. Hall, "Encyclopedia Briannica," 609 2018. [Online]. Available: https://www.britannica.com/topic/Intel. [Accessed 10 09 2018]

[2] Timetoast , "Timetoast," 2017. [Online]. Available: https://www.timetoast.com/timelines/linea-de-tiempo-cambiostecnologicos-tarjeta-madre. [Accessed 66 2018]

[3] HWlab, " HW-lab," 2017. [Online]. Available: https://hwlab.com/thermal-pads-forced-reality.html/4\#test. [Accessed 18 2018]

[4] D. Edwards, D. Glenn, F. Shaji, I. Sushumna and P. Frank, "Google Patents," 39 2003. [Online]. Available: https://patents.google.com/patent/US6444496B1/en?q=thermal $\& q=$ paste \&oq=thermal+paste. [Accessed 38 2018]

[5] A. Al-damook and F. Saleh, "Heat transfer and airflow characteristics enhancement of compact plate-pin fins heat sinks - a review," Propulsion and Power Research, vol. 7, no. 2, pp. 138-146, 1162018. 
[6] Intel, "Intel," $21 \quad 7 \quad 2017 . \quad$ [Online]. Available: https://www.intel.com/content/dam/support/us/en/documents/pr ocessors/sb/BXTS13A_Thermal_Solution_LGA2011V3.pdf. [Accessed 57 2018]

[7] D. Barragán, "Producción de entropía y ley de enfriamiento de Newton," Revista Ingeniería e Investigación, vol. 29, no. 2, pp. 88-93, 82009.

[8] M. Vogel, "Low Profile Heat Sink Cooling Technologies for Next Generation CPU Thermal Designs," 2005. [Online]. Available:

http://scholar.googleusercontent.com/scholar?q=cache:yIymrmc LzmcJ:scholar.google.com/+cpu+thermal+performance\&hl=es \&as_sdt=0,5. [Accessed 108 2018]

[9] D. Stone, "Smallbusiness Chron," 2018. [Online]. Available: https://smallbusiness.chron.com/thermal-compound-pc67419.html. [Accessed 128 2018]

[10] S. Burke, "Gamer Nexus," 1410 2015. [Online]. Available: https://www.gamersnexus.net/guides/2137-thermalpaste-typesconductivity-and-more. [Accessed 98 2018]

[11] J. Padilla, "WePC," 183 2018. [Online]. Available: https://www.wepc.com/tips/thermal-grease-paste-padsdifference/. [Accessed 19 2018]

[12] "Hardmaniacos," $2018 . \quad$ [Online]. Available: https://www.hardmaniacos.com/que-es-la-pasta-termica-quepasta-termica-utilizar/. [Accessed 66 2018]

[13] J. Wei, "Challenges in Cooling Design of CPU Packages for High-Performance Servers," Heat Transfer Engineering, vol. 29, no. 2, pp. 178-187, 2008.

[14] IC Innovation Cooling, "IC Innovation Cooling," 2017. [Online]. Available: https://www.innovationcooling.com/products/icdiamond/. [Accessed 139 2018]

[15] Arctic Silver, "Arctic Silver 5," 81 2018. [Online]. Available: http://www.arcticsilver.com/PDF/AS5_SDS.pdf. [Accessed 139 2018]

[16] Halnziye, "Halnziye," 9 2014. [Online]. Available: http://www.halnziye.net/download_detail/downloadsId=19.html . [Accessed 129 2018]

[17] Artic Silver Céramique 2, "Artic Silver Céramique 2," 812019. [Online]. Available: http://www.arcticsilver.com/PDF/CMQ2_SDS.pdf. [Accessed $1392018]$

[18] Halnziye, "Halnziye," Halnziye, 2017. [Online]. Available: http://www.halnziye.net/products_detail/productId=71.html. [Accessed 129 2018]

[19] Arctic Silver 2, "Arctic Silver ArctiClean 2," 76 2015. [Online]. Available: http://www.arcticsilver.com/PDF/ACN_1_SDS.pdf; http://www.arcticsilver.com/PDF/ACN_2_SDS.pdf. [Accessed $1292018]$

[20] R. Andrés, "Computer Hoy," 176 2016. [Online]. Available: https://computerhoy.com/paso-a-paso/hardware/como- controlar-temperatura-tu-ordenador-17819. [Accessed 159 2018]

[21] D. Cottino, J. Ghione and R. Martínez, "Users," in 200 Respuestas Hardware, Banfield, Gradi SA, 2008, p. 320.

[22] Build Computers, "Build Computers," 2016. [Online]. Available: http://www.buildcomputers.net/cpu-temperature.html. [Accessed 159 2018]

[23] Extech, "Extech," 2013. [Online]. Available: http://translate.extech.com/instruments/resources/manuals/EX8 40_UM-en.pdf. [Accessed 61 2018]

[24] Sabo, "Sabo Internacional," 2018. [Online]. Available: http://www.sabointernacional.com/es/?page_id=28. [Accessed 1192018 ]

[25] ASRock, "ASRock," 2009. [Online]. Available: ftp://asrock.cn/Manual/G41M-VS3.pdf. [Accessed 158 2018]

[26] Asus, "Asus," 7 2015. [Online]. Available: https://dlcdnets.asus.com/pub/ASUS/nb/X555UA/0409_E1046 5_X555U_A.pdf. [Accessed 109 2018]

[27] Acer, "Acer," 2016. [Online]. Available: https://globaldownload.acer.com/GDFiles/Document/User\%20Manual\%20 W10/User\%20Manual\%20W10_Acer_1.0_A_A.pdf?acerid=63 $6350239425113883 \&$ Step $1=$ NOTEBOOK\&Step2=ASPIRE $\& S$ tep3=ASPIRE\%20E5-

$553 \& O S=A L L \& L C=e s \& B C=A C E R \& S C=P A \_2$. [Accessed 11 9 2018]

[28] Open Hardware Monitor, "Open Hardware Monitor," 2018. [Online]. Available: https://openhardwaremonitor.org/. [Accessed 16 2018] 